\title{
Our experience with outpatient anesthesia in a patient with Huntington chorea
}

\begin{abstract}
Huntington chorea is a rare autosome dominant disorder. Men and women are equally affected as it is an autosomally transmitted. Symptoms occur in the third and fourth decade of life and manifest as involuntary choreiform movements, ataxia, and progressive mental deterioration. Patients are at higher risk for some intraoperative complications. Only a few case reports have been published reporting the anesthetic management of patients having Huntington's chorea and we would like to submit our experiences for this seldom case.
\end{abstract}

Keywords: huntington chorea, outpatient, propofol, dentistry
Volume 2 Issue 3 - 2015

\author{
Ozkan Onal,' Aynur Kaplan² \\ 'Department of Anesthesiology and Reanimation, Selcuk \\ University, Turkey \\ ${ }^{2}$ Department of Anesthesiology and Reanimation, Ankara \\ Tepebasi Teeth Hospital, Turkey
}

\begin{abstract}
Correspondence: Ozkan Onal, Department of Anesthesiology and Reanimation, Faculty of Medicine, Selcuk University, Konya,
\end{abstract} Turkey, Tel +905059052252, Email drozkanonal@selcuk.edu.tr

Received: March 30, 2015 | Published: April 24, 2015

\section{Introduction}

Huntington chorea is a rare autosome dominant disorder which occurs at the rate of 5-7 per 100.000 and involves nervous system. It was first defined in 1872 by George Huntington following his observations of families living in Huntington region of New York. Huntington's disease has the lowest spontaneous mutation rate among the other known genetic disorders. ${ }^{1}$ Men and women are equally affected as it is an autosomally transmitted. It is almost impossible for a person to develop the disease without an affected parent. The abnormal gene responsible is located in chromosome $4^{2}$ in which CAG triplet expansions in the Huntington disease gene on Chromosome 4 leads to the production of a polyglutamine gene product called Huntingtin. This protein aggregates in the cell cytoplasm and nuclei. ${ }^{3}$ Symptoms occur in the third and fourth decade of life and manifest as involuntary choreiform movements, ataxia, and progressive mental deterioration. ${ }^{4}$ Patients are at higher risk for some intraoperative complications (e.g., regurgitation and pulmonary aspiration), prolonged response to succinylcholine and thiopental, poor respiratory function, increased sensitivity to midazolam, and postoperative shivering resulting in rigid spasms. Only a few case reports have been published reporting the anesthetic management of patients having Huntington's chorea. ${ }^{3,5}$

Patients with this disease present with choreic movements, personality disorders and progressive decrease in cognitive functions between the ages of 35-40. In addition, in association with dysfunction in pharynx muscles, swallow and gag reflex may be impaired. The most marked motor symptom is dysphagia, which prevents food intake and gives rise to risk of aspiration. HD is an unusual neurodegenerative disorder that prior case reports suggest may have implications for the anesthesiologist. Administering anesthesia to a patient with Huntington chorea has many difficulties and there are very few case reports on this issue. The patients are under risk of pulmonary aspiration and have increased sensitivity to midazolam and there is prolonged response to sodium thiopental. Therefore, an anesthetic regimen that provides rapid recovery of airway reflexes is essential in order to avoid aspiration. Remifentanil is an opioid which undergoes metabolism by nonspecific blood and tissue esterases, unlike other members of this class of drugs that are metabolized by the liver.

It can be administered for a long duration, at a high dose without accumulation, avoiding the risks of prolonged respiratory impairment and enabling rapid recovery. ${ }^{3}$ In recent studies, sevoflurane has been shown to be effective in avoiding respiratory complications. ${ }^{6}$ In addition the use of total intravenous anesthesia (TIVA) with propofol has been advocated. ${ }^{7,8}$ to enable rapid restoration of airways reflexes. In this case report, a 68 year old female patient who was administered anesthesia successfully for tooth extraction is presented.

\section{Case report}

Operation for tooth extraction was planned for a 68 year old female patient who was diagnosed with Huntington chorea 25years ago. The patient who had severe disarthria, severe ataxia, presented to the operating room in restraints and exhibited advanced dementia and spontaneous vigorous choreoathetoid movements. Laboratory tests were normal except for hgb value of 10.7 and the patient had no history of hepatic, cardiovascular, pulmonary, endocrine renal or gastrointestinal tract disease. Lungs were normal upon examination, but in case extubation would not be possible, tooth extraction with sedation was planned. Intravenous access was almost impossible, because the patient tried to escape out of the bed and to get rid of her restraints. Mixture of oxygen and 3\% sevoflurane was given. Intravenous access was achieved after the patient became sedated. Following routine monitorization procedure, as a measure against aspiration, nasal oxygen cannula was inserted in upside down position and in induction, $10 \mathrm{mg}$ ranitab, $50 \mathrm{mcg}$ fentanyl and $50 \mathrm{mg}$ propofol iv were Administered. Muscle paralysis agents were not used and subsequently, $30 \mathrm{mg} / \mathrm{hr}$ propofol infusion and $0.25 \mathrm{mg} / \mathrm{hr}$ remifentanyl infusion was started. 13 teeth of the patients were extracted. And operation lasted around 45 minutes. No perioperative complications occurred. At the end of the procedure, propofol and remifentanyl infusions were discontinued. And the patients was transferred to recovery room. After staying there for 6 hours, the patient was taken to the clinic and 6 hours after the procedure the patients was discharged home.

\section{Discussion}

Huntington chorea is a neurodegenerative disease which is of importance for anesthesists. In Huntington chorea, there are many points that should be taken into account with regard to anesthesia. For example, premedication should not be carried out with 
metoklopropamide, since it induces increase in choreiform movements. Stewart stated that anticholinergics should be avoided as well, because there is a balance between dopamine and acethylcholine in striatum. ${ }^{9}$ If anticholinergics are administered, increase in choreiform movements may be observed..$^{10}$ Our patient was on antipsychotic, antidepressants, benzodiazepin and antiepileptic agents. And anesthetist should be aware of potential drug interaction with these drugs. Medications need to be specified as drug interaction is more important in such case. In patients such as ours, general anesthesia may lead to the exaggerated appearance of psychiatric symptoms such as postoperative agitation, chorea and psychosis. Maintenance of psychotropic drugs until the day of operation is important in these patients. There are publications reporting that in this patient group sodium thiopental may cause prolonged apnea. In our patient, we preferred propofol, as there was no report in the literature on its causing prolonged apnea. Kaufman $\&$ Erb. ${ }^{11}$ reported a case of a female patient aged 42years who was induced to a state of general anesthesia with propofol and then her sedation was maintained using propofol infusion supplemented with fentanyl. The patient reportedly opened her eyes after the infusion was discontinued. Another problem which is of concern from anesthesia is dysphagia, which occurs frequently in this patients group. As a consequence of dysphagia, which is the most marked motor symptom, food intake is prevented and risk of aspiration develops activation of airway reflexes after anesthesia is over, minimizes the risk of post-op aspiration. ${ }^{10}$ It has been proposed that these patients may be affected by non-depolarizing muscle relaxants, although the reported response to atracurium was normal. ${ }^{12,13}$ The incidence of decreased pseudocholin esterase activity is significantly higher in the patients with Huntington's disease than in normal population. ${ }^{14}$ This may explain the existing single report of prolonged response to succinylcholine. ${ }^{15}$. The primary goal in general anaesthesia for these patients is to provide airway protection and a rapid and safe recovery. The recently introduced inhalational agent propofol and remifentanil could eliminate the problem of prolonged recovery because of its favourable pharmacokinetic profile. ${ }^{15}$

\section{Conclusion}

Huntington chorea is a rare hereditary disease of nervous system. Experience with Huntington chorea is limited since it requires special drugs and techniques. Different anesthetic techniques have been recommended for Huntington's chorea, although the best anesthetic method remains elusive. ${ }^{3,5}$ Some practitioners recommend completely IV anesthesia methods that avoid. The patients from inhale potent drugs which can bring about postoperative shivering and precipitate generalized tonic spasms. Others have used inhaled anesthetics, including isoflurane, halothane and sevoflurane, without any problem. ${ }^{3}$ Main aim in anesthesia of these patients is the protection of airway and rapid and reliable recovery. We believe that in this patient group, propofol and remifentanil infusion fulfills these aims and can be used safely for outpatient anesthesia.

\section{Acknowledgements}

None.

\section{Conflicts of interest}

The authors declare that there are no conflicts of interest.

\section{Funding}

None.

\section{References}

1. Shaw M, Caro A. The mutation rate to Huntington's chorea. J Med Genet. 1982;19(3):161-167.

2. Gusella JF, Wexler NS, Conneally PM, et al. A polymorphic DNA marker genetically linked to Huntington's disease. Nature. 1983;306(5940):234-238.

3. MacPherson P, Harper I, MacDonald I. Propofol and Remifentanil Total Intravenous Anesthesia for a Patient with Huntington Disease. J Clin Anesth. 2004;16(7):537-538.

4. Bloch M, Adam S, Fuller A, et al. Diagnosis of Huntington disease: a model for the stages of psychological response based on experience of a predictive testing program. Am J Med Genet. 1993;47(3):368-374.

5. Davies DD. Abnormal response to anaesthesia in a case of Huntington's chorea. Br J Anaesth. 1966;38(6):490-491.

6. Nagele P, Hammerle AF. Sevoflurane and mivacurium in a patient with Huntington's chorea. Br J Anaesth. 2000;85(2):320-321.

7. Rodrigo MR. Huntington's chorea:midazolam, a suitable induction agent? Br J Anaesth. 1987;59(3):388-389.

8. Johnson MK, Heggie NM. Huntington's chorea. A role for the newer anaesthetic agents. Br J Anaesth. 1985;57(2):235-236.

9. Stewart JT. Huntington's disease. Am Fam Physician. 1988;37(5):105114.

10. Cangemi CF, Miller RJ. Huntington's Disease:Review and Anesthetic Case Management. Anesth Prog. 1998;45(4):150-153.

11. Blanloeil Y, Bigot A, Dixneuf B. Anaesthesia in Huntington's chorea. Anaesthesia. 1982;37(6):695-696.

12. Soar J, Matheson KH. A safe anaesthetic in Huntington's disease? Anaesthesia. 1993;48(8):743-744.

13. Lamont AMS. Brief report:Anaesthesia and Huntington's chorea. Anaesth Intensive Care. 1979;7(2):189190.

14. Gualandi W, Bonfati G (1968) A case of prolonged apnoea in Huntington's chorea. Acta Anaesthesiol. 1968;19(Suppl 6):235-238.

15. Anurag Yadava, Pradip KB, Rajnish KJ, et al. Anaesthesia and Huntington's Chorea. Indian J Anaesth. 2006;50(1):47-48. 